\title{
Formononetin inhibits osteosarcoma cell proliferation and promotes apoptosis by regulating the miR-214-3p/phosphatase and tensin homolog pathway
}

\author{
Kang $\mathrm{Li}^{1,2}$, Hongyuan Shen ${ }^{2}$, Minghui Lu ${ }^{3}$, Jiarong Chen ${ }^{2}$, Qingshui Yin ${ }^{2}$, Pingyue $\mathrm{Li}^{2}$ \\ ${ }^{1}$ Southern Medical University, Guangzhou 510515, China; ${ }^{2}$ Department of Orthopedics, General Hospital of Southern Theatre Command, \\ Guangzhou, China; ${ }^{3}$ The University of the South: Sewanee, TN, USA \\ Contributions: (I) Conception and design: K Li, Q Yin; (II) Administrative support: Q Yin; (III) Provision of study materials or patients: M Lu, P Li; \\ (IV) Collection and assembly of data: K Li, H Shen, M Lu; (V) Data analysis and interpretation: K Li, J Chen, M Lu; (VI) Manuscript writing: All \\ authors; (VII) Final approval of manuscript: All authors. \\ Correspondence to: Prof. Qingshui Yin. Department of Orthopedics, General Hospital of Southern Theatre Command, Liuhua Road, NO. 111 Street, \\ Guangzhou 510010, China. Email: 1k99199@163.com.
}

Background: Phytoestrogens have a similar molecular structure to estrogens which can produce either
estrogenic or anti-estrogenic effects. It is generally believed that phytoestrogens combine with the estrogen
receptor of osteosarcoma cells, affecting a variety of signal transduction pathways and cell metabolism,
resulting in altered cell proliferation, differentiation, apoptosis, invasion and migration ability. Formononetin
(FN) is the active ingredient of traditional Chinese medicine astragalus, angelica, and Pueraria lobate. Our
study aims to detect the role of FN on MG-63 cell viability and apoptosis through regulating phosphatase
and tensin homolog (PTEN) expression via MicroRNA-214-3p (miR-214-3p).

Methods: 3-(4,5-Dimethylthiazol-2-yl)-2,5-diphenyltetrazolium bromide (MTT) assay and Caspase 3 assay evaluated cell viability and apoptosis, respectively. Real-time quantitative polymerase chain reaction (qRTPCR) and western blot evaluated the mRNA and protein expressions, respectively. The binding site of miR214-3p/PTEN was detected via dual luciferase assay.

Results: FN suppressed cell viability and induced apoptosis, and decreased miR-214-3p level and promoted PTEN expression. PTEN was then regarded as a target of miR-214-3p, and FN improved PTEN level via inhibiting miR-214-3p. Further analysis showed that overexpressed miR-214-3p improved cell viability and suppressed apoptosis of MG-63 cells by inhibiting PTEN expression.

Conclusions: Finally, our results revealed that FN inhibited cell viability and induced apoptosis by regulating miR-214-3p. FN acted as a new treatment for MG-63 cells via increasing PTEN level by inhibiting the miR-214-3p level.

Keywords: PTEN; formononetin (FN); MicroRNA-214-3p; cell viability and apoptosis; osteosarcoma cell

Submitted May 19, 2020. Accepted for publication Jul 27, 2020.

doi: $10.21037 /$ tcr-20-2296

View this article at: http://dx.doi.org/10.21037/tcr-20-2296

\section{Introduction}

Osteosarcoma is a malignant tumor originating from mesenchymal tissue (1). The metaphysis of long bones in adolescents has a high malignancy, and lung metastasis can occur early, with a poor prognosis (2). At present, comprehensive treatment with chemotherapy and surgical resection as the core have been used to prolong the survival time of patients (3). However, because many mechanisms have not been resolved in the process of osteosarcoma development, osteosarcoma still has postoperative severe tumor recurrence and metastasis. Problems affecting the prognosis of patients (4). Most patients with osteosarcoma 
have tumor suppressor gene suppression, including retinoblastoma $(\mathrm{Rb})$ and phosphatase and tensin homolog (PTEN), high activation of the protein kinase B (AKT) pathway, and mutation of TP53 in some patients.

MicroRNAs (miRNAs) are non-lengths of 18 to $25 \mathrm{nt}$ and encode small molecule RNAs (5). miRNAs combine with the target gene mRNA through base-pairing to degrade the target mRNA or inhibit its translation (6). During the development of osteosarcoma, the expression of various miRNA changes and the upregulated or downregulated miRNAs are widely involved in the occurrence, development of osteosarcoma (7). After searching the GEO database, we found that miR-214-3p was significantly upregulated in osteosarcoma (8). Moreover, miR-214-3p is one of the miRNAs with oncogene properties that are important in the process of various cancers (9). The miR214-3p level is markedly increased in osteosarcoma $(9,10)$.

Formononetin $(\mathrm{FN})$ is the active ingredient of traditional Chinese medicine astragalus, angelica, and Pueraria lobate $(11,12)$. It belongs to isoflavone phytoestrogen and has pharmacological activities, including anti-oxidation, enhanced immunity, and anti-inflammatory $(13,14)$. Studies have found that $\mathrm{FN}$ can show satisfactory anticancer effects in ovarian cancer, colorectal cancer, and gastric cancer by suppressing cell viability and inducing apoptosis $(15,16)$. However, the effect and mechanism of FN on osteosarcoma cell proliferation and apoptosis are not apparent.

Therefore, this study hypothesized that the function of FN in MG-63 cells might be related to miR-214-3p/PTEN. The in vitro experiments were performed in MG-63 cells. Our experiment shows crucial opinions into the role of $\mathrm{FN}$ on the progress and development of osteosarcoma cells.

We present the following article in accordance with the MDAR reporting checklist (available at http://dx.doi. org/10.21037/tcr-20-2296).

\section{Methods}

\section{Cell culture and Transfection}

All media contained penicillin (100 IU/mL), 10\% (v/v), FBS and streptomycin $(100 \mathrm{mg} / \mathrm{mL})$. Inoculate MG-63 cell $\left(5 \times 10^{5}\right.$ cells/well $)$ in a 24 -well plate, and then incubate overnight. GenePharma (Shanghai, China) synthesized the miR-214-3p mimic or PTEN plasmid. Lipofectamine 2000 (Invitrogen, Carlsbad, CA, USA) transfected miR214-3p mimics (50 nM), PTEN plasmid (50 nM), and the corresponding negative controls in differentiated podocytes following with the manufacturer's instruction.

\section{Cell viability}

3-(4,5-Dimethylthiazol-2-yl)-2,5-diphenyltetrazolium bromide (MTT) solution measured the cell viability (Dojindo, Kumamoto, Japan). The MG-63 cells (2,000 cells/well) were seeded in 96-well plates. Then, the MG63 cell was incubated in 10\% MTT. And incubate in an incubator $\left(37^{\circ} \mathrm{C}, 5 \% \mathrm{CO}_{2}\right)$ for $2 \mathrm{~h}$. The OD value was evaluated at $450 \mathrm{~nm}$.

\section{Apoptosis}

A caspase-3 assay kit evaluated the apoptosis (Beyotime, Shanghai, China). Caspase 3 belongs to the CED-3 subfamily of the Caspase family and is a protease that plays an important role in the process of apoptosis (17). Caspase 3 Assay Kit is based on Caspase 3 which can catalyze the substrate acetyl-Asp-Glu-Val-Asp p-nitroanilide (Ac-DEVDpNA) to produce yellow p-nitroaniline (pNA), which has strong absorption around $405 \mathrm{~nm}$ The activity of Caspase 3 can be detected by measuring absorbance (yellow product is proportional to the activity of Caspase 3 enzyme). The MG-63 cells were treated with paraffin in 96-well plates for 90 minutes and passed through $80 \mu \mathrm{L}$ of reaction buffer (caspase-3 substrate, $2 \mathrm{mmol} / \mathrm{L}, 10 \mu \mathrm{L}$ ). Ten $\mu \mathrm{L}$ of cell lysate protein was incubated in each sample in an incubator $\left(37^{\circ} \mathrm{C}\right.$, $5 \% \mathrm{CO}_{2}$ ) for $2 \mathrm{~h}$. Used R\&D Systems' colorimetric assay kit (Thermo Scientific, USA) to measure samples at $405 \mathrm{~nm}$.

\section{Luciferase reporter assay}

The miR-214-3p putative binding site with wild type (WT) and mutant type (MUT) of PTEN 3'-UTR were combined and cloned into the pmirGLO dual-luciferase reporter vector (YouBio, Changsha, China). The reporter vectors (NC-mimic, miR-214-3p mimic, MUT, and WT 3'-UTR of PTEN) were co-transfected into HEK293T cells. After $48 \mathrm{~h}$, the activity of luciferase was evaluated by a dual luciferase assay (Promega, Madison, USA), standardized as Renilla luciferase.

\section{Real-time quantitative polymerase chain reaction (qRT- PCR)}

Trizol reagent (YouBio) extracted the total RNA of cell samples. Then, the SuperScript RT kit (YouBio) reverse 
transcribed $2 \mu \mathrm{g}$ of total RNA. ABI Prism7900 Sequence Detection System with SYBR Green PCR Master Mix (Applied Biosystems, USA) was used to perform qRT-PCR. Corresponding primers were synthesized by GenScript company (Nanjing, China) and showed as follow: PTENforward: AGAC CATA ACCC ACCA CAGC; PTENreverse: TACA CCAG TCCG TCCT TTCC. GAPDHforward: CGGC AAGT TCAA CGGC ACAG; GAPDHreverse: CGCC AGTA GACT CCAC GACA T. miR214-3p-forward: ACAG CAGG CACA GACA GGCA G; miR-214-3p-reverse: GTGC AGGG TCCG AGGT ATTC. 5S-forward: GATC TCGG AAGC TAAG CAGG; 5S-reverse: TGGT GCAG GGTC CGAG GTAT. Among them, GAPDH and 5S rRNA were applied as internal references of above-mentioned genes and miR-214-3p respectively. Calculating targeted gene expression following the $2^{-\triangle \Delta C T}$ method (18). GAPDH and U6 snRNA are used as reference genes, respectively.

\section{Western blot}

Cells were lysed with pre-chilled radioImmunoprecipitation Assay (RIPA) buffer (addition of cocktail and phosphatase inhibitor), $30 \mu \mathrm{g} / \mathrm{sample}$ was taken for sodium salt-Polyacrylamide gel electrophoresis (SDS-PAGE) electrophoresis, and transferred into a polyvinylidene fluoride (PVDF) membrane $(0.45 \mu \mathrm{m}), 1$ $\times$ Tris- Buffered Saline Tween20 (TBST) The diluted 2\% Bovine Serum Albumin (BSA) was blocked, and the primary antibody (PTEN, Caspase 3, Caspase 9, Bcl-2) was cultured $\left(4{ }^{\circ} \mathrm{C}, 12 \mathrm{~h}\right)$. The goat Anti-Mouse IgG-HRP $(1: 3,000)$ (Solarbio) for $\beta$-actin is cultured $\left(37^{\circ} \mathrm{C}, 1 \mathrm{~h}\right)$. After 4 times of TBST washing, the images were added dropwise.

\section{Statistical analysis}

The GraphPad 8.0 was used for statistical analysis. All data were expressed as an independent experiment three times and shown as mean \pm standard deviation (SD). Statistical analysis following with the ANOVA and the $\mathrm{P}<0.05$ of data were considered significant.

\section{Results}

FN can down-regulate miR-214-3p expression and promote PTEN levels

To evaluate the inhibitory role of $\mathrm{FN}$ on the cell viability of MG-63 cell treatment of FN (0, 15, 30, and $45 \mu \mathrm{M})$ for $48 \mathrm{~h}$ and then evaluated by MTT assay. As compared to the control group, as the FN concentration gradually increased, the cell viability was markedly inhibited (Figure 1A). After MG-63 cell treatment of FN, a definite increase in the number of apoptotic cells with $45 \mu \mathrm{M} \mathrm{FN}$ was found $(\mathrm{P}<0.05)$ (Figure $1 B)$. The data showed that FN-induced apoptosis with dose-dependent. Then, qRTPCR analysis on miR-214-3p and PTEN expressions after treatment of $\mathrm{FN}(0,15,30$, and $45 \mu \mathrm{M})$ were also detected, and results exhibited the miR-214-3p level was markedly reduced and PTEN level was markedly improved by FN treatment with dose-dependent (Figure1C). Differently, the PTEN level was improved after the treatment of FN (Figure 1D).

\section{miR-214-3p4-3p can bind PTEN and inbibit PTEN expression}

PTEN was whether predicted to the miR-214-3p by the TargetScan database, and the synthetic 3'-UTR fragment of PTEN was cloned into pmirGLO to construct WT or MUT dual-luciferase reporter plasmids (Figure 2A). Moreover, Figure $2 B$ exhibited that the activity of luciferase was reduced after treatment of miR-214-3p mimic and WT-PTEN 3'-UTR vector as compared to that treatment of miR-214-3p mimic and MUT-PTEN 3'-UTR. MG63 cells were treated with miR-214-3p mimic to verify the relationship, and the transfection efficiency was determined. The miR-214-3p level was markedly increased after the treatment of miR-214-3p mimic (Figure 2C). However, the PTEN level was significantly decreased by miR-214$3 \mathrm{p}$ mimic (Figure 2D). These data showed that miR-214-3p could bind PTEN and inhibit the PTEN level.

\section{miR-214-3p improves MG-63 cell viability and inhibits apoptosis by down-regulating PTEN expression}

To further determine whether PTEN was involved in the roles of miR-214-3p on the cell viability and apoptosis, MG-63 cell was treated with miR-214-3p mimic vector alone, or miR-214-3p mimic + PTEN vector for $24 \mathrm{~h}$. The proliferation was inhibited, and the apoptosis was improved by the treatment of miR-214-3p mimic + PTEN vector as compared to that treatment of miR-214-3p mimic vector alone (Figure $3 A, B$ ). Moreover, the data of western blot exhibited that the Caspase 3, Caspase 9, PTEN protein expression was upregulated in the miR-214-3p mimic + 

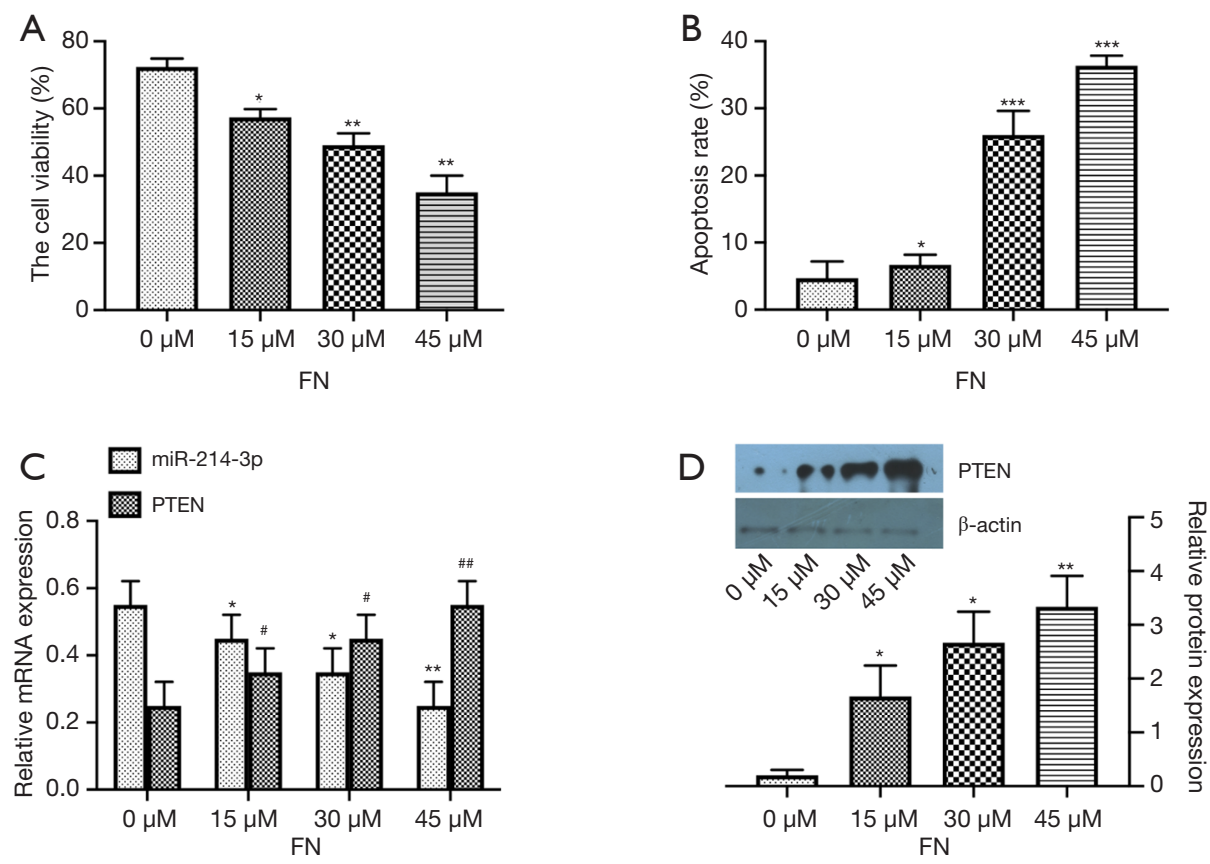

Figure 1 Effect of formononetin (FN) on the miR-214-3p and PTEN expression. (A) The cell viability was evaluated by MTT assay. (B) Caspase 3 kit was used to evaluate the apoptosis rate of cells. (C) The miR-214-3p and PTEN mRNA expressions were evaluated by realtime quantitative polymerase chain reaction (qRT-PCR). (D) Western blotting evaluated the $\mathrm{PTEN}$ level. ${ }^{*} \mathrm{P}<0.05,{ }^{* *} \mathrm{P}<0.01,{ }^{* * *} \mathrm{P}<0.001$ vs. FN $(0 \mu M) ;{ }^{\#} \mathrm{P}<0.05,{ }^{\#} \mathrm{P}<0.01$ vs. FN $(0 \mu \mathrm{M}) \mathrm{PTEN}$.

A hsa-miR-214-3p: 3'-UGACGGACAGACACGGACGACA-5'
PTEN 3'-UTR:
I I I। II । position 1014-1020: 5'-UUUUCAAUCAUAAUACCUGCUGU-3' position 3416-3422: 5'-UCAGUUUUUUUUUUUCCUGCUGU-3'

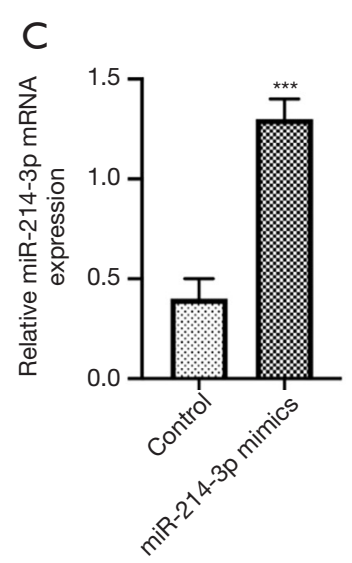

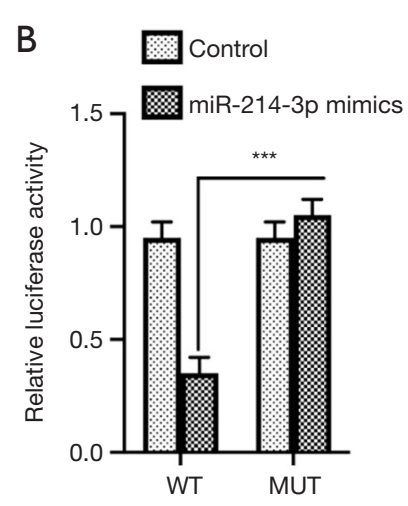

Figure 2 miR-214-3p binds phosphatase and tensin homolog (PTEN) and inhibit PTEN expression. (A) TargetScan predicts miR-2143p targets the PTEN. (B) the miR-214-3p binding site on the 3'-UTR of PTEN mRNA was evaluated, and the activity of luciferase was examined in HEK-293T. (C) Real-time quantitative polymerase chain reaction (qRT-PCR) evaluated the miR-214-3p mRNA expression. (D) Western blot evaluated the PTEN level. ${ }^{* *} \mathrm{P}<0.01$, ${ }^{* * *} \mathrm{P}<0.001$ vs. control. 

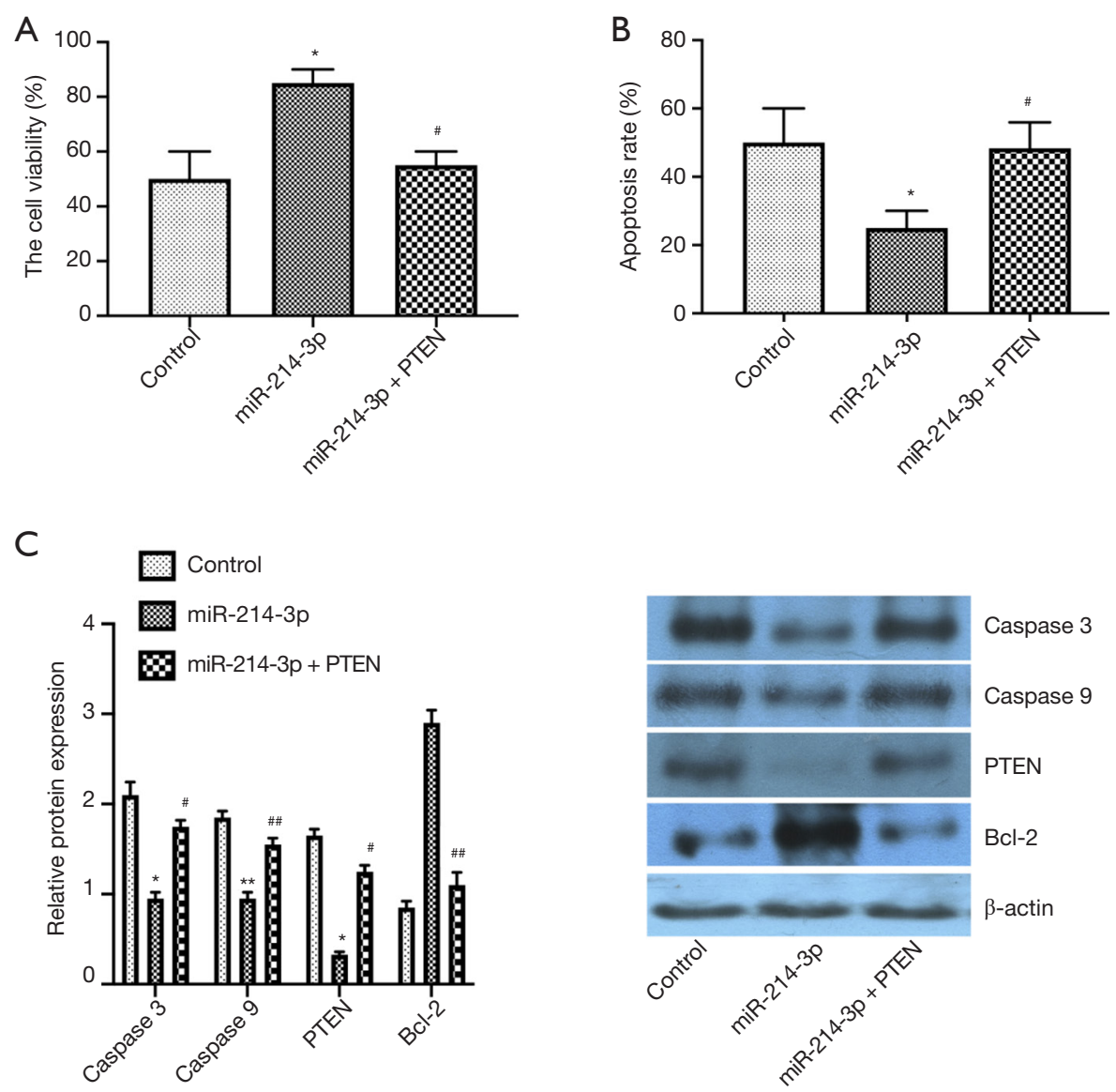

Figure 3 miR-214-3p promotes MG-63 cell viability and inhibits apoptosis through down-regulating phosphatase and tensin homolog (PTEN) expression. (A) The cell viability was evaluated by MTT assay. (B) Caspase 3 kit was used to evaluate the apoptosis rate of cells. (C) Caspase 3, Caspase 9, PTEN, Bcl-2 levels were evaluated by western blotting. ${ }^{*} \mathrm{P}<0.05,{ }^{* *} \mathrm{P}<0.01$ vs. control; ${ }^{\#} \mathrm{P}<0.05$, ${ }^{\# \#} \mathrm{P}<0.01$ vs. miR-2143 p mimics.

PTEN group as compared to the miR-214-3p mimic group, while the expression of Bcl-2 was down-regulated (Figure 3C). The above data showed that overexpressed miR-214-3p improved cell viability and inhibited apoptosis of MG-63 cells, at least partially, by inhibiting PTEN expression.

\section{FN inhibits osteosarcoma cell viability and induces apoptosis by down-regulating miR-214-3p}

Whether the miR-214-3p-mediated cell viability and apoptosis were modulated by the FN, MG-63 cells were treated with $\mathrm{FN}(30 \mu \mathrm{M})$, miR-214-3p mimic, or FN $(30 \mu M)+$ miR-214-3p mimic. qRT-PCR exhibited that FN treatment markedly reduced the miR-214-3p expression was as compared to the miR-214-3p mimic group (Figure 4A). MTT assay exhibited that the suppression role of FN in cell proliferation was attenuated by miR-214-3p, revealing that the cell viability in $\mathrm{FN}(30 \mu \mathrm{M})+$ miR-214-3p mimic group was markedly improved as compared to that in $\mathrm{FN}(30 \mu \mathrm{M})$ group (Figure 4B). Moreover, the Caspase 3 assay results suggested that miR-214-3p suppressed $\mathrm{FN}$-induced cell apoptosis (Figure 4C). Moreover, the results of the western blot showed that the Caspase 3, Caspase 9, PTEN protein expressions were upregulated in the miR-214-3p mimic group as compared to the miR-214-3p mimic group, while the expression of Bcl-2 was down-regulated (Figure $4 D$ ). The above results showed that the anti-tumor role of $\mathrm{FN}$ in MG-63 might be partially through regulating miR-214-3p.

\section{Discussion}

$\mathrm{FN}$ has the effects of regulating estrogen, inflammation, 
A

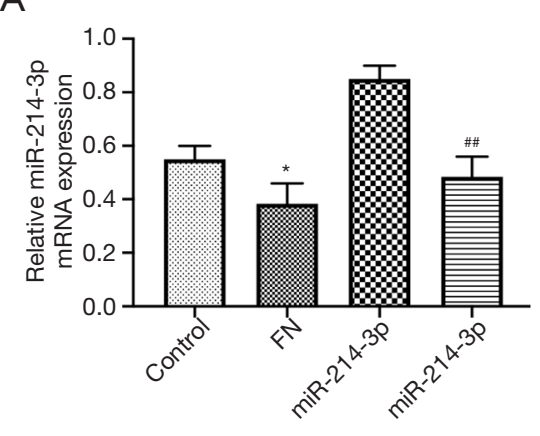

B

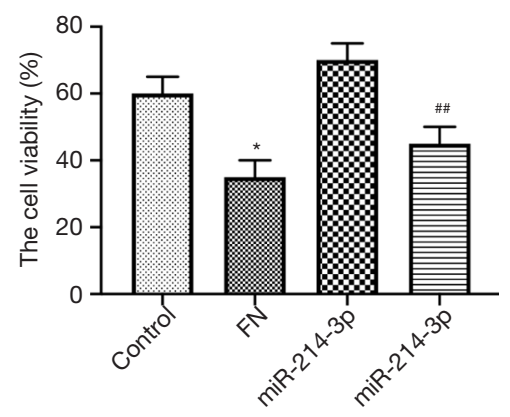

C

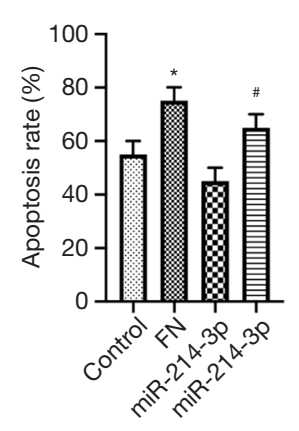

$\mathrm{D}$

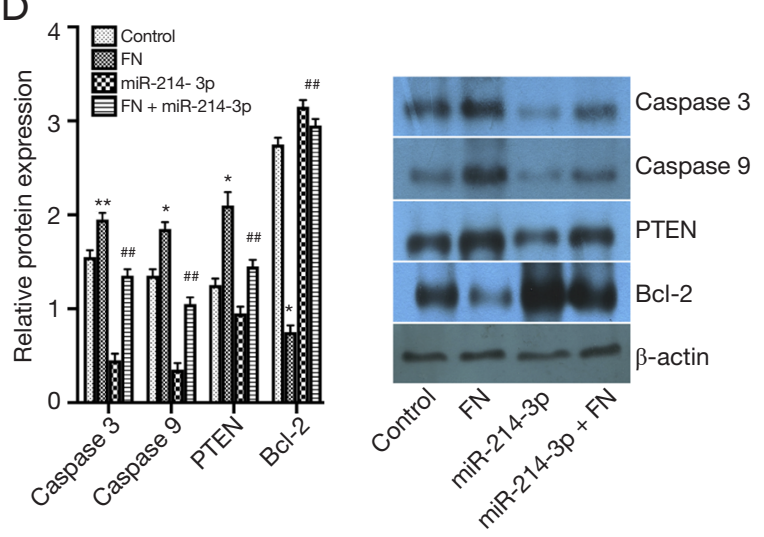

Figure 4 Formononetin (FN) inhibits osteosarcoma cell viability and induces apoptosis by decreasing miR-214-3p. (A) The miR-214-3p mRNA level was evaluated by real-time quantitative polymerase chain reaction (qRT-PCR). (B) MTT assay evaluated cell viability. (C) The apoptosis rate of cells was evaluated by the Caspase 3 kit. (D) Caspase 3, Caspase 9, phosphatase and tensin homolog (PTEN), Bcl-2 levels were evaluated by western blot. ${ }^{*} \mathrm{P}<0.05,{ }^{* *} \mathrm{P}<0.01$ vs. control; ${ }^{\#} \mathrm{P}<0.05,{ }^{\# \#} \mathrm{P}<0.01$ vs. miR-214-3p mimics.

and lowering blood pressure. Also, FN plays an anti-tumor effect in various ways (19). Wang and other studies pointed out that FN suppressed the cell viability of colon tumor cells via inhibiting the cell cycle at the G0/G1 phase by suppressing the Cyclin D1 protein level; also, FN inhibits the cell viability of colon tumor cells (20). The role of FN is also related to the up-regulation of miR-149 expression and suppression of PI3K/AKT and STAT3 signaling activation (20). Hu and others found that FN suppressed the cell viability of osteosarcoma cells in vivo and in vitro, and promoted the Bax expression, an apoptosis protein bax, by decreasing the miR-375 expression and the inhibitory protein Bcl-2 expression that induces apoptosis (21). In the present study, FN suppressed the cell viability and induced apoptosis, and decreased miR-214-3p level and promoted PTEN level.

miR-214-3p is one of the miRNAs with oncogene properties that are important in the process of various cancers (9). The miR-214-3p level is markedly increased in osteosarcoma $(9,10)$. Liver cancer has been shown to have oncogene capabilities. For example, in liver cancer, miR-214$3 p$ bonds directly with Wnt3a to improve the proliferation $(22,23)$. Recent reports have found miR-214-3p regulates Wnt3a in osteosarcoma (9). miR-214-3p participates in the growth and metastasis of cancers through targeted regulation of the critical tumor suppressor gene PTEN (24). PTEN is a crucial tumor inhibitor gene and is closely related to various tumors (25). PTEN is the most critical upstream molecule in the protein kinase B (AKT) pathway, and it plays a role in inhibiting AKT phosphorylation through dephosphorylation/proximal interphalangeal joints 3 (PIP3) (26). Disturbance of the phosphatidylinositol 3 kinase (PI3K)/AKT signaling is widespread in many cancers, which inhibit apoptosis, promote cell viability, affect synthesis and sugar metabolism (27). PTEN was predicted to be a binding site of miR-214-3p by TargetScan database, and miR214-3p levels were significantly increased in MG-63 cells transfecting with miR-214-3p mimic. However, the PTEN 
level was significantly decreased by a miR-214-3p mimic. Moreover, the activity of luciferase was treatment with miR214-3p mimic and WT-PTEN 3'-UTR vector as compared to that treatment of miR-214-3p mimic and MUT-PTEN 3'-UTR.

Classical apoptosis pathways include endogenous mitochondrial cytochrome release signaling and exogenous death receptor signaling. Existing research shows that these two pathways are not independent, but interrelated and intertwined, and these two pathways will eventually activate and wither end-of-life effect enzyme caspase 3 (28-30). The Bcl-2 and Bax expressions were changed markedly through western blot and qRT-PCR. The mitochondrialmediated apoptosis pathway is regarded as a critical center of apoptosis and is only regulated by the proteins of the Bcl2 family (31). Bcl-2 is one of the most critical inhibitors of apoptosis, and Bax promoted apoptosis, a critical factor that determines whether cells survive or die (32). In the current study, overexpressed miR-214-3p improved cell viability and suppressed apoptosis of MG-63 by inhibiting PTEN expression. Moreover, FN inhibited proliferation and induced apoptosis of MG-63 by regulating miR-214-3p.

\section{Conclusions}

Our results showed that FN might be acting as a novel treatment for MG-63 through the up-regulation of PTEN expression via miR-214-3p.

\section{Acknowledgments}

Funding: This work was supported by the National Natural Science Foundation of China (81871808); the Science and Technology Planning Project of Guangdong Province (2017B030314139); Basic research project of military application (CLB18J037); Guangzhou Science and technology plan project (201707010015) and Science and Technology Planning Project of Guangzhou (20190401315).

\section{Footnote}

Reporting Checklist: The authors have completed the MDAR reporting checklist. Available at http://dx.doi.org/10.21037/ tcr-20-2296

Data Sharing Statement: Available at http://dx.doi. org/10.21037/tcr-20-2296
Conflicts of Interest: All authors have completed the ICMJE uniform disclosure form (available at http://dx.doi. org/10.21037/tcr-20-2296). The authors have no conflicts of interest to declare.

Ethical Statement: The authors are accountable for all aspects of the work in ensuring that questions related to the accuracy or integrity of any part of the work are appropriately investigated and resolved.

Open Access Statement: This is an Open Access article distributed in accordance with the Creative Commons Attribution-NonCommercial-NoDerivs 4.0 International License (CC BY-NC-ND 4.0), which permits the noncommercial replication and distribution of the article with the strict proviso that no changes or edits are made and the original work is properly cited (including links to both the formal publication through the relevant DOI and the license). See: https://creativecommons.org/licenses/by-nc-nd/4.0/.

\section{References}

1. Shi J, Zhao C, Liu X, et al. The regulatory role of aberrant methylation of microRNA-34a promoter CpGs in osteosarcoma. Transl Cancer Res 2019;8:2328-38.

2. Zwaga T, Bovee JVMG, Kroon HM. Osteosarcoma of the Femur with Skip, Lymph Node, and Lung Metastases. Radiographics 2008;28:277-83.

3. Yu P, Wen J, Wang J, et al. Establishment and characterization of a novel human osteosarcoma cell line for spontaneous pulmonary metastasis research in vivo. Ann Transl Med 2019;7:573.

4. Mirabello L, Troisi R, Savage SA. Osteosarcoma incidence and survival rates from 1973 to 2004: Data from the Surveillance, Epidemiology, and End Results Program. Cancer 2009;115:1531-43.

5. Hackenberg $M$, Gustafson $P$, Langridge $P$, et al. Differential expression of microRNAs and other small RNAs in barley between water and drought conditions. Plant Biotechnol J 2015;13:2-13.

6. Catalanotto C, Cogoni C, Zardo G. MicroRNA in Control of Gene Expression: An Overview of Nuclear Functions. Int J Mol Sci 2016;17:1712-29.

7. Gulyaeva LF, Kushlinskiy NE. Regulatory mechanisms of microRNA expression. J Transl Med 2016;14:143-52.

8. Tian R, Xie X, Han J, et al. miR-199a-3p negatively regulates the progression of osteosarcoma through 
targeting AXL. Am J Cancer Res 2014;4:738-50.

9. Xu C, He T, Li Z, et al. Regulation of HOXA11-AS/miR214-3p/EZH2 axis on the growth, migration and invasion of glioma cells. Biomed Pharmacother 2017;95:1504-13.

10. Zhao X, Wang Q, Lin F, et al. RNA Sequencing of Osteosarcoma Gene Expression Profile Revealed that miR-214-3p Facilitates Osteosarcoma Cell Proliferation via Targeting Ubiquinol-Cytochrome c Reductase Core Protein 1 (UQCRC1). Med Sci Monitor 2019;25:4982-91.

11. Ong SKL, Shanmugam MK, Fan L, et al. Focus on Formononetin: Anticancer Potential and Molecular Targets. Cancers 2019;11:611-34.

12. Wang S, Wu X, Tan M, et al. Fighting fire with fire: Poisonous Chinese herbal medicine for cancer therapy. J Ethnopharmacol 2012;140:33-45.

13. Park S, Bazer FW, Lim W, et al. The O-methylated isoflavone, formononetin, inhibits human ovarian cancer cell proliferation by sub G0/G1 cell phase arrest through PI3K/AKT and ERK1/2 inactivation. J Cell Biochem 2018;119:7377-87.

14. He X, Dixon RA. Genetic Manipulation of Isoflavone 7-O-Methyltransferase Enhances Biosynthesis of 4'-O-Methylated Isoflavonoid Phytoalexins and Disease Resistance in Alfalfa. Plant Cell 2000;12:1689-702.

15. Nicastro HL, Ross SA, Milner JA. Garlic and Onions: Their Cancer Prevention Properties. Cancer Prev Res 2015;8:181-9.

16. Rahman K. Garlic and aging: new insights into an old remedy. Ageing Res Rev 2003;2:39-56.

17. Wright ME, Han DKM, Carter L, et al. Caspase-3 inhibits growth in Saccharomyces cerevisiae without causing cell death. Febs Lett 1999;446:9-14.

18. Livak KJ, Schmittgen TD. Analysis of relative gene expression data using real-time quantitative PCR and the 2- $\Delta \Delta$ CT method. Methods 2001;25:402-8.

19. Liu Z, Wu H, Wei Z, et al. TRPM8: a potential target for cancer treatment. J Cancer Res Clin 2016;142:1871-81.

20. Wang AL, Li Y, Zhao Q, et al. Formononetin inhibits colon carcinoma cell growth and invasion by microRNA-149-mediated EphB3 downregulation and inhibition of PI3K/AKT and STAT3 signaling pathways. Mol Med Rep 2018;17:7721-9.

21. Hu W, Xiao Z. Formononetin induces apoptosis of human osteosarcoma cell line U2OS by regulating the expression of Bcl-2, Bax and MiR-375 in vitro and in vivo. Cell Physiol Biochem 2015;37:933-9.

22. Zhao C, Sun W, Zhang P, et al. miR-214 promotes osteoclastogenesis by targeting Pten/PI3k/Akt pathway. RNA Biol 2015;12:343-53.

23. Zhu XB, Zhang ZC, Han GS, et al. Overexpression of miR-214 promotes the progression of human osteosarcoma by regulating the $W n t / \beta$-catenin signaling pathway. Mol Med Rep 2017;15:1884-92.

24. Han LC, Wang H, Niu FL, et al. Effect miR-214$3 \mathrm{p}$ on proliferation and apoptosis of breast cancer cells by targeting survivin protein. Eur Rev Med Pharmaco 2019;23:7469-74.

25. Ishii N, Maier D, Merlo A, et al. Frequent Co-Alterations of TP53, p16/CDKN2A, p14ARF, PTEN Tumor Suppressor Genes in Human Glioma Cell Lines. Brain Pathol 1999;9:469-79.

26. McCubrey JA, Steelman LS, Abrams SL, et al. Roles of the RAF/MEK/ERK and PI3K/PTEN/AKT pathways in malignant transformation and drug resistance. Adv Enzyme Regul 2006;46:249-79.

27. Fritz V, Fajas L. Metabolism and proliferation share common regulatory pathways in cancer cells. Oncogene 2010;29:4369-77.

28. Qiu M, Chen L, Tan G, et al. JS-K promotes apoptosis by inducing ROS production in human prostate cancer cells. Oncol Lett 2017;13:1137-42.

29. Lee JC, Shin EA, Kim B, et al. Auraptene induces apoptosis via myeloid cell leukemia 1-mediated activation of caspases in PC3 and DU145 prostate cancer cells. Phytother Res 2017;31:891-8.

30. Kim YS, Kim SK, Park SJ. Apoptotic effect of demethoxyfumitremorgin $\mathrm{C}$ from marine fungus Aspergillus fumigatus on PC3 human prostate cancer cells. Chem-biol Interact 2017;269:18-24.

31. Davids MS, Letai A. Targeting the B-cell lymphoma/ leukemia 2 family in cancer. J Clin Oncol 2012;30:3127-35.

32. Kang MH, Reynolds CP. Bcl-2 inhibitors: targeting mitochondrial apoptotic pathways in cancer therapy. Clin Cancer Res 2009;15:1126-32.

(English Language Editor: J. Chapnick)

Cite this article as: Li K, Shen H, Lu M, Chen J, Yin Q, Li P. Formononetin inhibits osteosarcoma cell proliferation and promotes apoptosis by regulating the miR-214-3p/ PTEN pathway. Transl Cancer Res 2020;9(8):4914-4921. doi: $10.21037 /$ tcr-20-2296 